\title{
GENETIC ROBUSTIFICATION OF DIGITAL TRAJECTORY-TRACKING CONTROLLERS FOR ROBOTIC MANIPULATORS
}

\author{
B Porter and C Allaoui \\ Intelligent Machinery Division \\ Research Institute for Design, Manufacture, and Marketing \\ University of Salford \\ Salford M5 4WT
}

\begin{abstract}
In this paper, genetic algorithms are used to robustify digital multivariable PID controllers for robotic manipulators. This process of robustification is effected by using genetic algorithms to determine the optimal set of controller tuning parameters for typical trajectory- tracking tasks. This use of genetic algorithms is illustrated by the design of a digital trajectory-tracking controller for a typical three-degree-of-freedom robotic manipulator.
\end{abstract}

\section{INTRODUCTION}

The design of effective digital trajectory-tracking controllers for robotic manipulators constitutes a very difficult problem in control engineering, because such manipulators are non-linear multivariable plants with time-varying parameters. However, it was shown by Porter and Abidin [1] that the design of such trajectory-tracking controllers for robotic manipulators can be readily effected by using the methodologies of Porter et al [2] for the design of fast-sampling error-actuated digital multivariable PID controllers. These methodologies of Porter et al [2] are remarkable since, even in the case of complex linear and non-linear plants, only the first and second Markov parameters of the linear components of such plants are required in the design equations. It was also shown by Porter et al [2] that these design equations can be further simplified by introducing the step-response matrices of linear multivariable plants instead of the first and second Markov parameters. This simplification, in turn, made it possible for Porter and Manganas [3] to show that these fast-sampling error-actuated digital controllers can be readily rendered adaptive since step-response matrices can be directly identified in real time by conventional recursive estimation techniques.

However, such relatively complicated adaptive fast-sampling error-actuated digital PID controllers need be used only if the underlying non-adaptive controllers are insufficiently robust in the face of plant-parameter variations. The robustness characteristics of such non-adaptive controllers were therefore established by Porter and Abidin [1] in the case of completely irregular multivariable plants, ie, plants (like most robotic manipulators) with null first Markov parameters and full-rank second Markov parameters. It was thus shown by Porter and Abidin [1] that the plant-parameter variations tolerable by fast-sampling error-actuated digital PID controllers can be expressed very simply in terms of the step-response matrices of the nominal and the actual plants. These general results were illustrated by examining the robustness of a fast-sampling error-actuated digital PD controller for the three-degree-of-freedom robotic manipulator previously investigated by Petropoulakis [4].

However, although the theoretical robustness results of Porter and Abidin [1] greatly facilitate the design of digital trajectory-tracking controllers for robotic manipulators, these theoretical results are valid only asymptotically (ie, as the sampling frequency of the digital PID controller becomes infinite). In practice, of course, the sampling frequencies of such digital controllers must remain finite; but it has so far proved impossible to obtain theoretical non-asymptotic robustness results for error-actuated digital multivariable PID controllers with finite sampling frequencies. Therefore, in this paper, genetic algorithms [5][6] are used to robustify digital multivariable PID controllers for robotic manipulators for typical trajectory-tracking tasks. This process of robustification is effected by using genetic algorithms to determine the optimal quadruple of controller tuning parameters for such trajectory-tracking tasks (which include sudden changes of payload). This use of genetic algorithms is illustrated by the design of a robustified digital trajectory-tracking PID controller for the robotic manipulator previously investigated by Petropoulakis [4]. These genetic design results are the natural extension to robotic control problems of the previously obtained non-robotic results of Porter and Jones [7] and Porter, Mohamed, and Jones [8].

\section{GENETIC DESIGN PROCEDURE}

The methodology of Porter et al [2] for the design of error-actuated digital PID controllers relates to linear multivariable plants governed on the continuous-time set $T=[0,+\infty)$ by state and output equations of the respective forms

and

$$
\begin{array}{lll}
\dot{x}(t) & =A x(t)+B u(t) & \ldots(t) \\
y(t)=C x(t) & \ldots(2)
\end{array}
$$

In equations (1) and (2), $x(t) \in R^{n}, u(t) \in R^{1}, y(t) \in R^{1}$, $A \in R^{n \times n}, B \in R^{n \times l}$, and $C \in R^{k \times n}$. In addition, it is assumed that the plants under control are completely irregular so that

$$
\operatorname{rank} C B=0
$$




$$
M(\theta) \theta+v(\theta, \theta)+g(\theta)=u \quad \ldots(14 a)
$$

and

$$
y=f(\theta),
$$

where $\theta \in R^{3}$ is the vector of joint angles, $u \in R^{3}$ is the vector of joint torques, $y \in R^{3}$ is the positional vector of the end effector in Cartesian space, $M(\theta) \in R^{3 \times 3}$ is the inertia matrix, $v(\theta, \theta) \in R^{3}$ is the vector of centrifugal and coriolis torques, $f(\theta) \in R^{3}$ is the vector of direct kinematic relationships, and $g(\theta) \in R^{3}$ is the vector of gravitational torques. The numerical values of the inertial and kinematic parameters for a typical three-degree-of-freedom robotic manipulator are given by Petropoulakis [4].

In the neighbourhood of any operating point in task space (or joint space), this manipulator is a completely irregular sixth-order linear multivariable plant with three inputs and three outputs. In order to illustrate the genetic robustification procedure, it is therefore instructive to design a trajectory-tracking digital PID controller for the linearised dynamics of the manipulator corresponding to the end-effector position $(0,0.45,0) \mathrm{m}$. This controller is then used while the end effector of the manipulator is caused to track straight-line trajectories betwen the following two points:

$$
\begin{array}{lll}
I & (-0.5,0,-0.2) m \\
I I & (-0.4,0.3,0) m \\
I I I & (0.3,0.3,0.3) m \\
I V & (0.3,0.3,0.3) m \\
V I & (-0.45,0.35,0) m
\end{array}
$$

These transitions are effected with 'trapezoidal' acceleration, cruise, and deceleration profiles in the following times:

$$
\begin{array}{lll}
I \rightarrow I I & 1.5 s \\
I I \rightarrow I I I & 2 s & , \\
I I I \rightarrow I V & 0.5 s & , \\
I V \rightarrow V & 2.5 s & ; \\
V \rightarrow V I & 0.5 s &
\end{array}
$$

In addition, after the initial transition $I \rightarrow I I$, the manipulator grasps an additional payload of $5 \mathrm{~kg}$. In view of the intrinsic non-linearity of the robotic manipulator and the sudden variation in payload, this sequence of tracking tasks constitutes a formidable test of robustness for the non-adaptive digital PID controller.

However, the genetic robustification of this controller for this trajectory-tracking task can be readily effected for any measure of tracking performance specified by the designer. Thus, for example, let it be desired to determine the controller quadruple $\{\alpha, \sigma, \rho, \delta\}$ so as to minimise the cost function

$$
\Gamma=\int_{o}^{\tau}\|e(t)\| d t
$$

where $\tau$ is the duration of the tracking task, $e(t) \in R^{3}$ is the trajectory-tracking error vector in Cartesian space, and $\|\cdot\|$

denotes the Euclidean norm. The results of the genetic robustification procedure in this case are shown in Figures 1 and 2 over 50 generations for a population size $N=30$, a crossover probability $p_{c}=0.6$, and a mutation probability $p_{m}=0.008$. In
Figure 1, the associated best-of-generation and generation-average values of the cost function $\Gamma$ are plotted against generation number, whilst, in Figure 2, the associated best-of-generation values of the controller parameters $\alpha, \sigma, \rho$, and $\delta$ are plotted against generation number. It is evident from these figures that the optimal quadruple of controller parameters is $\{\alpha, \sigma, \rho, \delta\}=\{0.231,0.838,20.95,0.015\}$ for which the corresponding value of the cost function is $\Gamma=\{0.01179\}$. The time-domain behaviour of $\|e(t)\|$ for this robustified trajectory-tracking digital PID controller is shown in Figure 3.

It is interesting to note that the quadruple of controller parameters for the non-genetically robustified controller of Porter and Abidin $[1]$ is $\{\alpha, \sigma, \rho, \delta\}=\{0.1,0.7,1,0.01\}$ for which the corresponding cost function is $\Gamma=\{0.01613\}$. It is thus evident that the performance of the genetically designed trajectory-tracking controller is superior to that of its non-genetically designed counterpart.

\section{CONCLUSION}

In this paper, genetic algorithms have been used to robustify digital multivariable PID controllers for robotic manipulators for typical trajectory-tracking tasks. This process of robustification has been effected by using genetic algorithms to determine the optimal set of controller tuning parameters for such trajectory-tracking tasks (which include sudden changes of payload). This use of genetic algorithms has been illustrated by the design of a digital trajectory-tracking controller for the robotic manipulator previously investigated non-genetically by Petropoulakis [4] and by Porter and Abidin [1].

\section{REFERENCES}

[1] B Porter and Z Abidin, "Robustness characteristics of fast-sampling digital set-point tracking PID controllers for completely irregular linear multivariable plants", Proc 4th Inst MC Symposium on Application of Multivariable System Techniques, Bradford, England, April 1990.

[2] B Porter, A Manganas, and T Manganas, "Design of fast non-interacting digital flight-mode control systems for high-performance aircraft", Proc AIAA Guidance, Navigation, and Control Conference, Snowmass, USA, August 1985.

[3] B Porter and A Manganas, "Design of adaptive fast-sampling digital set-point tracking controllers incorporating recursive step-response matrix identifiers for unstable multivariable plants", Proc IFAC Workshop on Adaptive Control of Chemical Processes, Frankfurt, Germany, October 1985.

[4] L Petropoulakis, "Design of digital trajectory tracking systems for robotic manipulators", $\mathrm{PhD}$ Thesis, University of Salford, 1986.

[5] J H Holland, "Adaptation in Natural and Artificial Systems", University of Michigan Press, 1975.

[6] DE Goldberg, "Genetic Algorithms in Search, Optimization, and Machine Learning", Addison-Wesley, 1989.

[7] B Porter and A H Jones, "Genetic tuning of digital PID controllers", Electronics Letters, Vol 28, p 843, 1992.

[8] B Porter, S S Mohamed, and A H Jones, "Genetic tuning of digital PID controllers for linear multivariable plants", Proc European Control Conference, Groningen, The Netherlands, June/July 1993. 


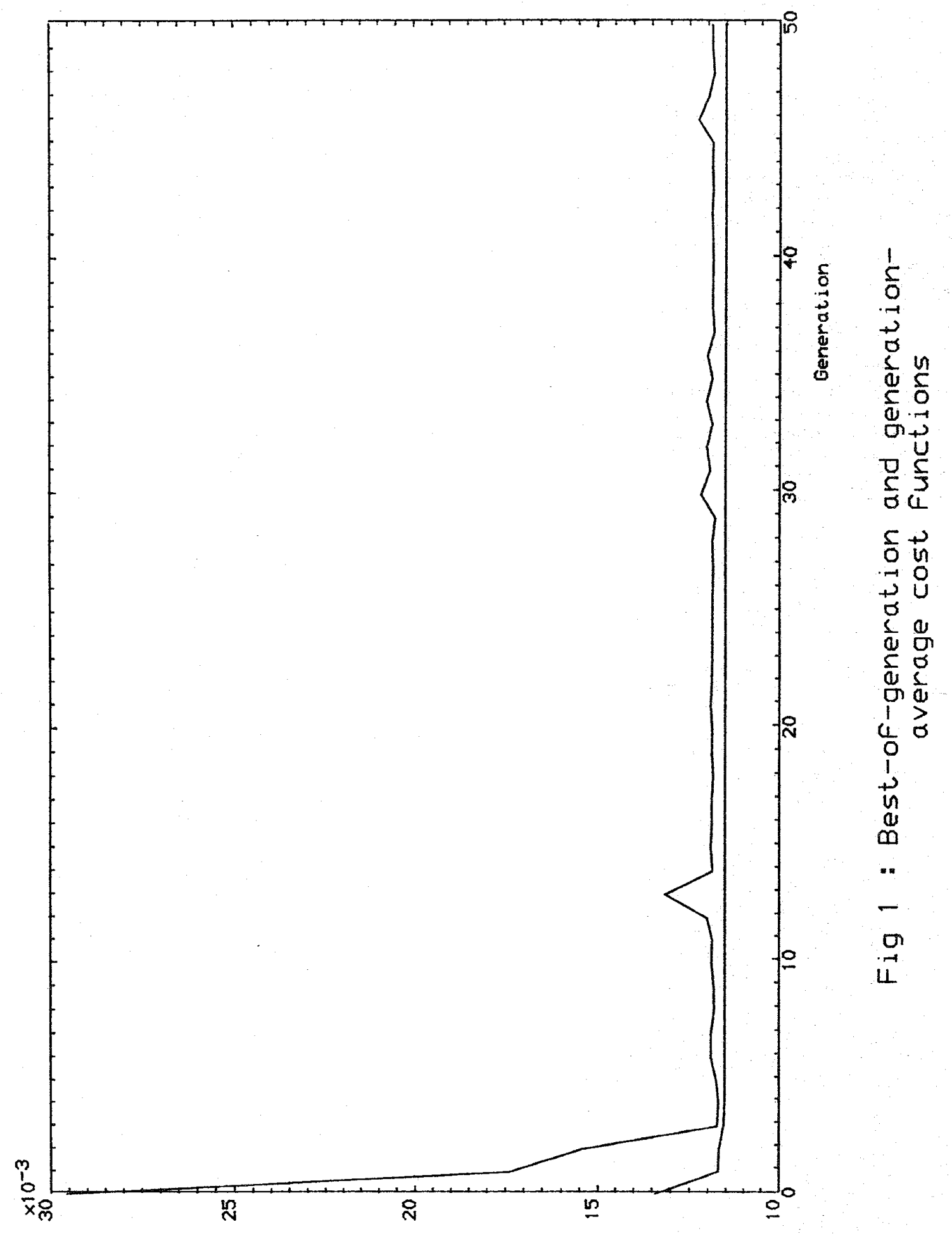

efonany 7508 

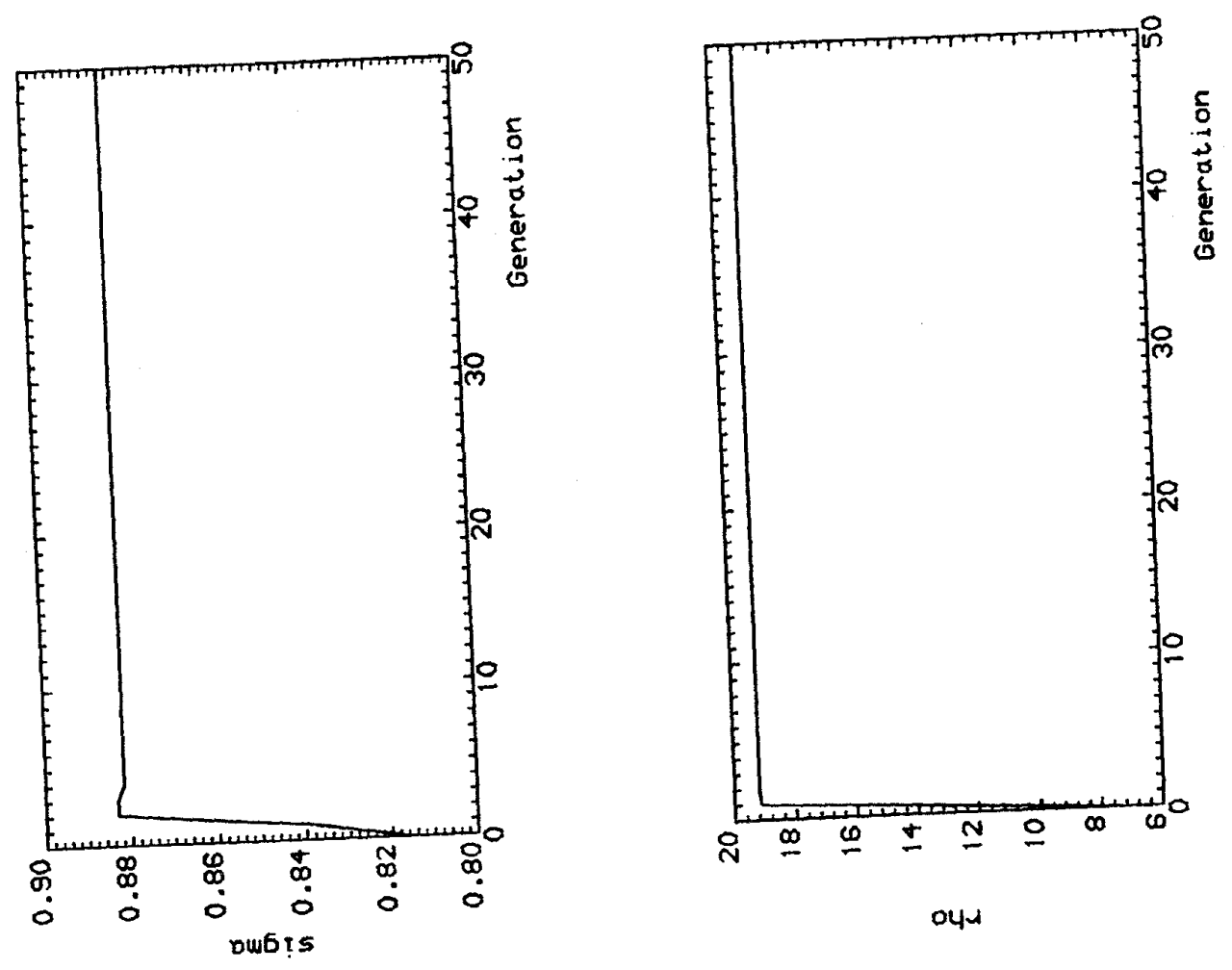

$n$
1
0
+3
0
0
5
0
0
5
0
0
0
1
5
5
0
0
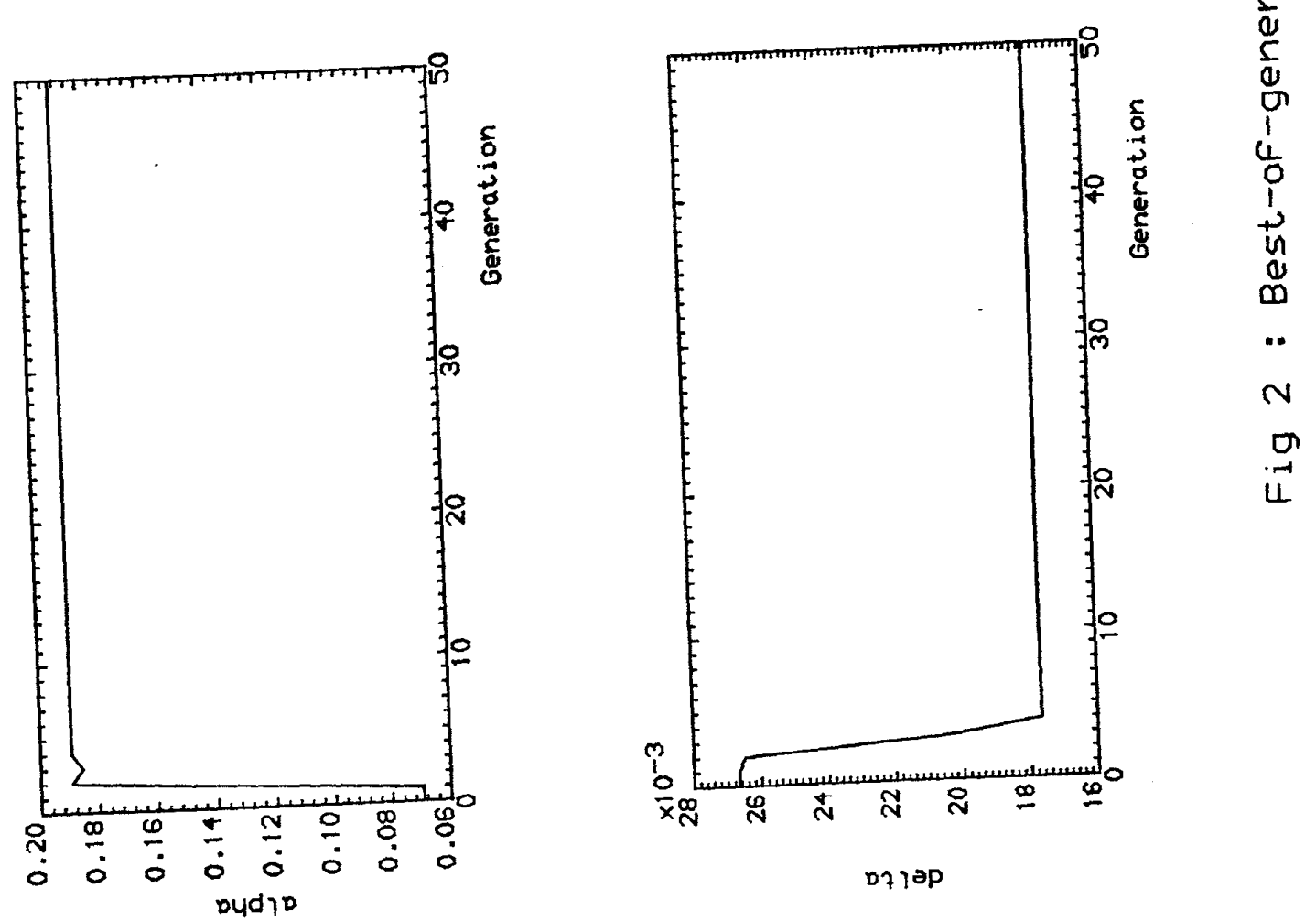


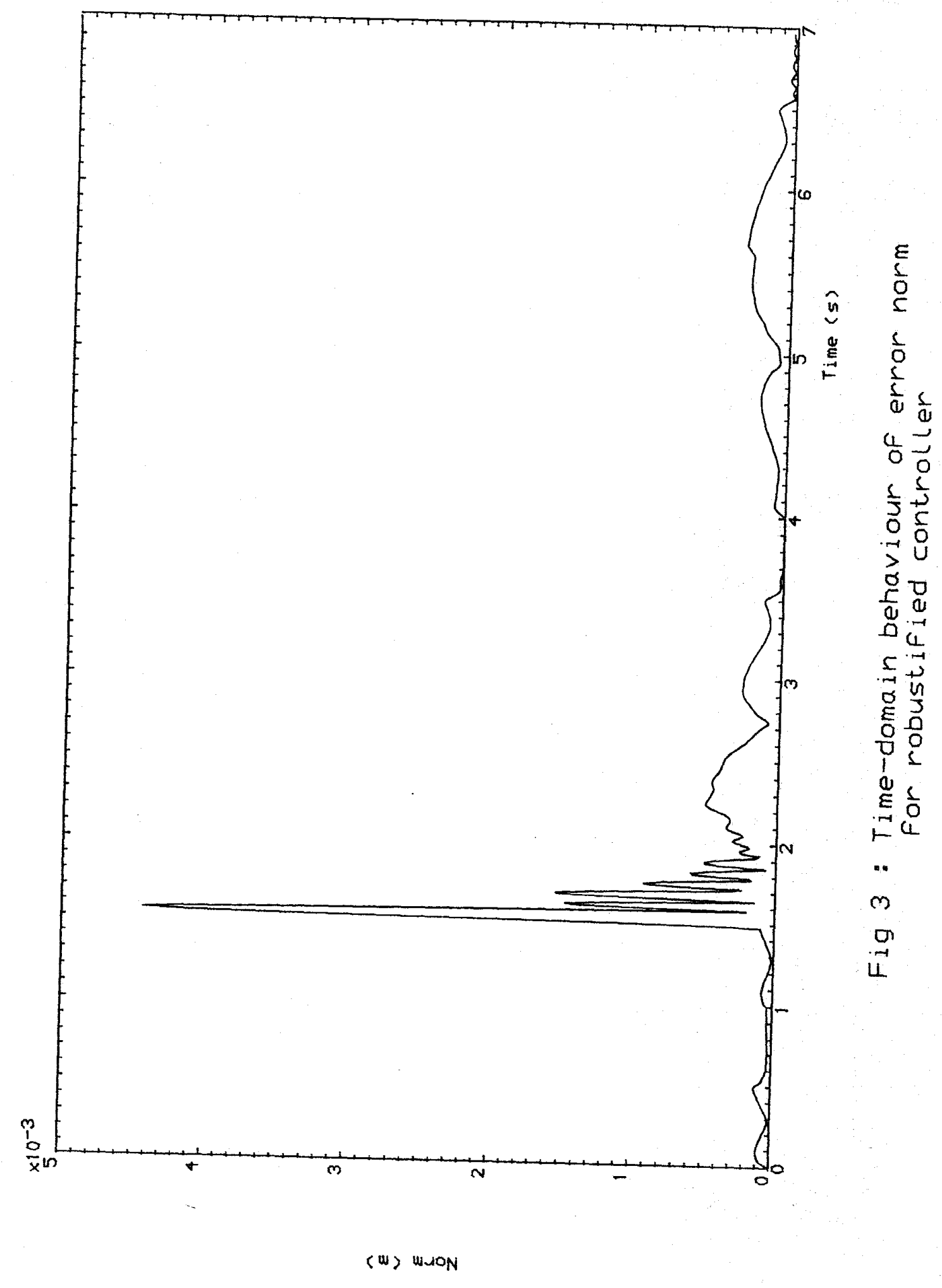

4426 
and

$$
\operatorname{rank} C A B=l
$$

The digital PID controllers under consideration are governed on the discrete-time set $T_{T}=\{0, T, 2 T, \ldots\}$ by control-law equations of the form

$$
u(k T)=K_{1} r(k T)+K_{2} z(k T)
$$

In equation (5), $T \in R^{+}$is the sampling period, $K_{1} \in R^{\mid \times l}$ and $K_{2} \in R^{\perp \times I}$ are the proportional and integral controller matrices, and the vectors $r(k T) \in R^{l}$ and $z(k T) \in R^{l}$ are generated in accordance with the difference equations

$$
\begin{aligned}
& s\{(k+1) T\}=-\alpha s(k T)+e(k T), \\
& \ldots(6 a) \\
& r(k T)=-\frac{2}{T}(1+\alpha) D s(k T)+\left(I_{l}+\frac{2}{T} D\right) e(k T),
\end{aligned}
$$

and

$$
z\{(k+1) T\}=z(k T)+\operatorname{Tr}(k T) \quad \ldots \quad \ldots(7)
$$

Moreover, in equations (6), $\alpha \in(-1,+1), s\left(k T \in R^{1}, e(k T)=\right.$ $v(k T)-y(k T) \in R^{1}$ is the error vector, $v(k T) \in R^{l}$ is the set-point command vector, and the derivative matrix $D \in R^{\ltimes \times l}$ is such that

$$
\operatorname{rank} D=l
$$

In order to investigate the robustness characteristics of error-actuated digital PD controllers for completely irregular multivariable plants, Porter and Abidin [1] expressed the design equations for the proportional and integral controller matrices in equation (5) in the forms

$$
K_{1}=T \bar{H}^{-1}(T) \Sigma\left(T I_{l}+2 D\right)^{-1} \in R^{\lfloor\rfloor J}
$$

and

$$
K_{2}=\rho T \bar{H}^{-1}(T) \Sigma\left(T I_{l}+2 D\right)^{-1} \in R^{I \times l} .
$$

In equations (12) and (13),

$$
\Sigma=\sigma I_{l} \quad\left(\sigma \in R^{+}\right)
$$

is the positive diagonal tuning matrix,

$$
D=\delta I_{l} \quad\left(\delta \in R^{+}\right)
$$

is the positive diagonal derivative matrix, and $\rho \in R^{+}$is the ratio of integral to proportional action. In addition, in equations (9) and $(10)$

$$
\bar{H}(T)=\int_{0}^{T} \bar{C} e^{\bar{A} t} \bar{B} d t \in R^{l \times l}
$$

is the step-respone matrix of the nominal open-loop plant with state-space triple $(\bar{A}, \bar{B}, \bar{C})$ which is used for design purposes in obtaining the controller for the actual open-loop plant with state space triple $(A, B, C)$ governed by equations (1) and (2). Indeed, by using the explicit expressions for $K_{1}$ and $K_{2}$ given by equations (9) and (10), Porter and Abidin [1] elucidated the required asymptotic robustness characteristics of the digital PID controllers governed by equations (5), (6), and (7).

However, although the robustness theorems thus established by Porter and Abidin [1] are very elegant, these results are restricted to the asymptotic case of fast-sampling error-actuated digital PD controllers for which the sampling frequency $f=1 / T \rightarrow \infty$. Furthermore, it transpires that these asymptotic results involve only the pair $\{\alpha, \sigma\}$ of design parameters rather than the complete quadruple $\{\alpha, \sigma, \rho, \delta\}$ of design parameters involved in the controller design equations (9) and (10). In practice, of course, the sampling frequencies of digital PID controllers must remain finite. It is therefore necessary to consider the following general robustness problem for the non-asymptotic case:

In the case of finite sampling frequency, determine the set of actual plants with state-space triple $(A, B, C)$ tolerable by digital PID controllers designed for the nominal plant with state-space triple $(\bar{A}, \bar{B}, \bar{C})$ and characterised by the quadruple $\{\alpha, \sigma, \rho, \delta\}$ of controller design parameters.

However, it has so far proved impossible to solve this problem theoretically. This motivates the use of genetic algorithms to solve the non-asymptotic robustness problem for digital PID controllers. In the context of robotic trajectory-tracking controllers, genetic algorithms can accordingly be used to solve the following version of the robustness problem:

In the case of finite sampling frequency, determine the quadruple $\{\alpha, \sigma, \rho, \delta\}$ of controller design parameters such that optimal trajectory-tracking behaviour is obtained when a given robotic manipulator is controlled so as to track a given trajectory.

It is evident that the solution of this problem will provide an optimal quadruple $\{\alpha, \sigma, \rho, \delta\}$ which is dependent upon the given manipulator, the given task, and the measure of trajectorytracking accuracy used in the optimisation procedure.

However, in order to use genetic algorithms to solve this problem, it is necessary only to encode the quadruple $\{\alpha, \sigma, \rho, \delta\}$ of controller design parameters in accordance with a system of concatenated, multi-parameter, mapped, fixed-point coding [6]. Thus, each quadruple $\{\alpha, \sigma, \rho, \delta\}$ of controller parameters is represented by a string of binary digits. Then, following any choice of an initial generation of such strings, successive generations of strings can be rapidly obtained using the basic genetic operations of selection, crossover, and mutation [6]. In particular, these operations ensure that the successive generations of error-actuated digital PID controllers thus produced by the genetic algorithm tend to exhibit improving trajectory-tracking behaviour in respect of any measure of the accuracy of such behaviour specified by the designer for any given robotic manipulator.

\section{ILLUSTRATIVE EXAMPLE}

This general approach to genetic robustification can be conveniently illustrated by designing a trajectory-tracking digital PID controller for the three-degree-of-freedom robotic manipulator previously investigated by Petropoulakis [4]. In this case, the manipulator is governed on $T=[0,+\infty)$ by state and output equations of the respective forms 